\title{
Absolute Position Measurement in a Gas Time Projection Chamber via Transverse Diffusion of Drift Charge
}

\author{
P. M. Lewis, S. E. Vahsen*, I. S. Seong, M. T. Hedges, I. Jaegle, T. N. Thorpe \\ University of Hawaii, 2505 Correa Road, Honolulu, HI 96822, USA
}

\begin{abstract}
Time Projection Chambers (TPCs) with charge readout via micro pattern gaseous detectors can provide detailed measurements of charge density distributions. We here report on measurements of alpha particle tracks, using a TPC where the drift charge is amplified with Gas Electron Multipliers and detected with a pixel ASIC. We find that by measuring the 3-D topology of drift charge and fitting for its transverse diffusion, we obtain the absolute position of tracks in the drift direction. For example, we obtain a precision of $\sim 1 \mathrm{~cm}$ for $0.8 \mathrm{~cm}$-long alpha track segments. To our knowledge this is the first demonstration of such a measurement in a gas TPC. This technique has several attractive features: it does not require knowledge of the initial specific ionization, is robust against bias from diffuse charge below detection threshold, and is also robust against high charge densities that saturate the detector response.
\end{abstract}

Keywords: TPC, GEM, pixel, diffusion, directional, neutron, dark matter

\section{Introduction}

Since their introduction in the 1970s, Time Projection Chambers (TPCs) [1] have found a wide range of applications in particle physics and beyond. Similar to bubble chambers, TPCs allow 3-D reconstruction of ionization distributions in large volumes. Traditionally, one limitation of the TPC has been that ${ }^{33}$ unless the time when ionization occurred is known, the detector only measures relative, not absolute position in the drift direc- ${ }^{34}$ tion, $z$. Recently, however, Snowden-Ifft of the Directional Re- ${ }^{35}$ coil Identification from Tracks (DRIFT) collaboration demon- ${ }^{36}$ strated the first absolute position measurement in a negative ion ${ }^{37}$ drift gas TPC by using a gas mixture with multiple charge car- ${ }^{38}$ riers [2]. In prior work, the DRIFT collaboration had used ${ }^{39}$ longitudinal diffusion to estimate $z$ and veto backgrounds [3]. ${ }^{40}$ However, the resolution was limited, so that the veto severely ${ }^{4}$ truncated the sensitive volume. Highlighting the importance of ${ }^{42}$ measuring $z$, the improved technique with multiple charge car- ${ }^{43}$ riers increased the DRIFT fiducial region from 5-10\% to $75 \%{ }^{44}$ of the detector volume, and reduced their background rate by at ${ }^{45}$ least two orders of magnitude, allowing background free oper- ${ }^{46}$ ation for the first time [4].

Here we show how a gas TPC with a high-density pixel ${ }^{48}$ readout plane is capable of measuring the absolute $z$ position ${ }^{49}$ of tracks with a different method, by measuring the detailed ${ }^{50}$ ionization density distribution of the diffused track after drift. ${ }^{51}$ As a bonus, the charge density distribution also provides suf- ${ }^{52}$ ficient information to measure the initial specific ionization of ${ }^{53}$ the track, automatically correcting for highly diffuse charge that ${ }^{54}$

${ }^{*}$ Corresponding author. Tel.: +1 8089562985 .

Email address: sevahsen@hawaii.edu (S. E. Vahsen) g9 goes undetected due to the threshold of the detector, and for high charge densities outside the dynamic range of the detector. The ionization measurement will be published separately, and is not explored further in this work.

\section{Detector and principle of operation}

We use a prototype directional fast-neutron detector that is an evolution of the miniature TPC described in Ref. [5]. Details on the detector used here will be published separately [7]. In brief, the detector has a sensitive volume of $2.0 \times 1.68 \times 15 \mathrm{~cm}^{3}$ in $x \times y \times z$, filled with a 70:30 mixture of $\mathrm{He}: \mathrm{CO}_{2}$ gas at atmospheric pressure. A field cage creates a uniform drift field of $530 \mathrm{~V} / \mathrm{cm}$ in the sensitive volume. Ionization deposited in the sensitive volume drifts through a distance $z$ then is amplified with two Gas Electron Multipliers (GEMs) [8], and finally is collected with an ATLAS FE-I4B pixel ASIC [9] (or "chip"), which digitizes the charge signal. The ATLAS FE-I4B was developed for the recent upgrade of the ATLAS Pixel Detector, a vertex detector used in the first run of the CERN Large Hadron Collider.

We use the chip to collect drifting charge, with high resolution in space and time. Charge is measured in units of time over threshold (ToT), which is not linear in collected charge and is based on a $40 \mathrm{MHz}$ clock. Spatially, the chip plane is segmented into 26,880 pixels of $50 \times 250 \mu \mathrm{m}^{2}$, arranged in a rectangular grid of 336 rows and 80 columns, dense enough to resolve the $\sim 1 \mathrm{~mm}$ typical width of drifted electron clouds in our TPC. We define the coordinate system so that the chip occupies the $x-y$ plane, perpendicular to the electric field $(+z)$ and the electron drift direction $(-z)$. Time bins are $25 \mathrm{~ns}$, which translates into a $z$ quantization of $250 \mu \mathrm{m}$ with our drift field. 


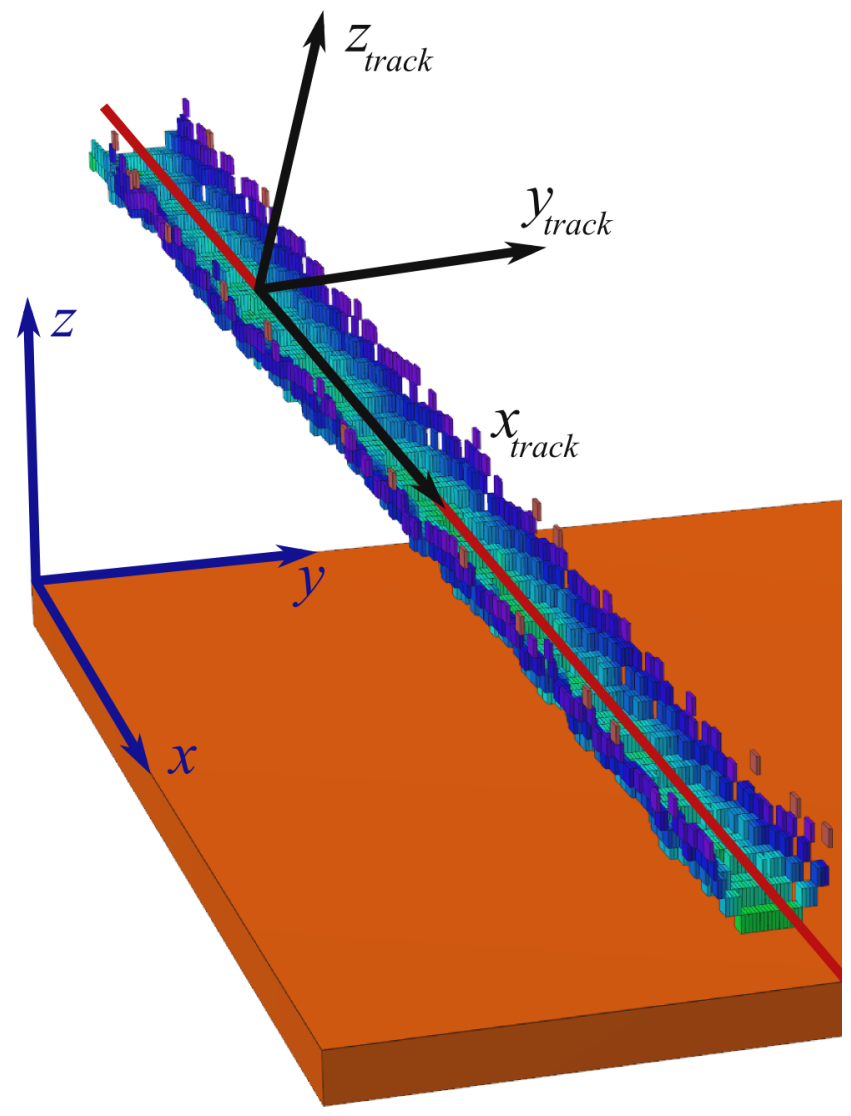

Figure 1: A typical alpha track from the dataset used in this analysis. Each box is located in 3-D space according to the $x, y$ and $z$ coordinates of the first threshold-crossing for the pixel under it. ToT is encoded by brightness (color online), with higher ToT (lighter) in the bottom of the barrel and low ToT (darker) at the high edges. Both coordinate systems used in this paper are drawn: the chip-based Cartesian coordinate system with $z$ pointing anti-parallel to the drift direction and the track coordinate system in which $y_{\text {track }}$ is the lateral displacement of a hit from the best-fit line (red online) to the track shell. The track angles $\theta$ and $\phi$ are the polar angle with respect to the $z$ axis, and the azimuthal angle between the track's $x-y$ projection and the $x$ axis, respectively. These angles define the rotations between the two coordinate systems. The absolute origin of the track coordinate system along the track fit line is arbitrary. The large plane below the track represents the bottom of the sensitive volume.

During normal operation of our TPC we keep three Polonium-210 alpha sources mounted on the outside of the field cage at $z=3.3 \mathrm{~cm}, 7.5 \mathrm{~cm}$, and $11.1 \mathrm{~cm}$. We use these in-situ sources to calibrate and monitor the detector in a number of ways in real-time. We discovered that these sources can also be used to calibrate the absolute $z$ scale of the TPC, a technique ${ }_{75}$ we demonstrate here.

\subsection{Charge cloud detection}

As a drifting charge cloud arrives at a pixel, the 4-bit ToT 79 counter starts once the accumulated charge reaches threshold 80 and continues to count for as long as the pixel is over thresh- 81 old. The ToT then represents the integral over time (and thus ${ }_{82}$ $z$ ) of the charge collected by each pixel. The chip output data 83 then contain no charge structure in $z$, only the relative threshold- 84 crossing time of the charge cloud from pixel to pixel in units 85

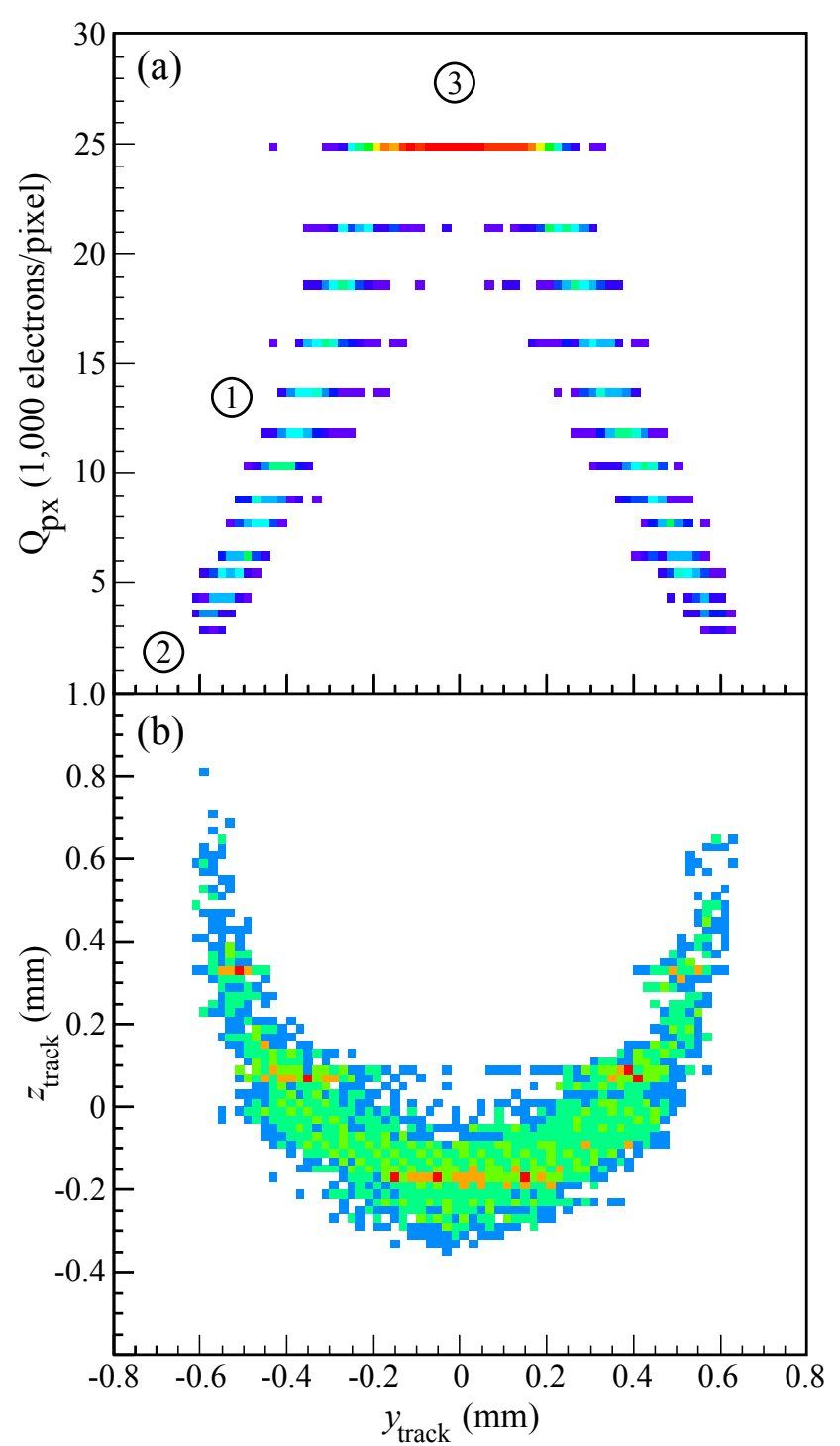

Figure 2: Corrected pixel charge $\left(Q_{p x}\right)$ profile (a) and shell coordinates (b) for a single horizontal track from the near alpha source. Label 1 of the profile plot (a) corresponds to the Gaussian, label 2 to the threshold, and label 3 to the saturation regions of the profile. The U-shaped shell in plot (b) is very roughly the bottom half of the track in space, described in Section 2.1. These plots are two-dimensional histograms where the counts per bin are encoded by brightness (color online): The outside points of each distribution (blue online) have the lowest count number, the center points (yellow and red online) have higher count numbers.

of $25 \mathrm{~ns}$. Some charge is lost, such as below-threshold charge at the edge of a track or charge that arrives after a pixel has fallen below threshold. The chip therefore provides four dimensions of track topology information for each pixel, as visualized in Fig. 1, namely: $x, y$, and $z$ spatial coordinates of the first threshold crossing for each pixel, plus ToT. The spatial coordinates define the charge "shell", which can be visualized semi-accurately as the bottom half of a track.

In our operation mode, the chip issues a trigger to the readout electronics when a pixel crosses threshold. The readout board then retrieves pixel data from pixels which crossed threshold during a fixed time window for a fixed time offset from the re- 


\section{Physics model} rive the expected variation of track topology with $z$. Specif- $-^{110}$ ically, we consider a line of ionization charge with an initial ${ }^{11}$ delta-function charge cross-section. The constituent charges ${ }^{112}$ diffuse in space in a 3-D random walk over time, giving a charge ${ }^{113}$ profile which is Gaussian in cross-section with a constant inte- ${ }^{114}$ gral and a width that is proportional to the square root of time, ${ }^{115}$ $\sigma_{z}(t) \propto \sqrt{t}$. In the presence of a linear potential, the charge ${ }^{116}$ line drifts to the detector plane at a constant velocity, implying ${ }^{117}$ that the width of the charge distribution is also proportional to ${ }^{118}$ the square root of absolute $z$, or $\sigma_{z}(z)=B \sqrt{z}$. There is a fixed $z$-independent width $A$ associated with the resolution of reading ${ }^{119}$ out a track after no drift, leading to an expected relationship be-- ${ }_{120}$ tween the width of the charge distribution and absolute $z$ given $_{121}$ by the quadrature sum of both terms [5]:

$$
\sigma(z)=\sqrt{A^{2}+B^{2} z}
$$

We use the chip's built-in capacitors to inject known charges into each pixel to measure an empirical correspondence benot a consistent measure of charge density along a track. For an inclined track, the density will appear higher as the track is horizontal track has $\theta=90^{\circ}$ ). We define a new quantity, the corrected pixel charge $Q_{p x}$, which includes a geometric correction and can be thought of as the expected charge collected in a pixel (in electrons) if the track was horizontal:

$$
Q_{p x}(\mathrm{ToT})=N_{e}(\mathrm{ToT}) \sin \theta
$$

Our model does not include two minor effects: first, re-126 absorption of drift electrons with gas impurities such as oxy-127 gen, which results in a linear decrease in the total charge of the ${ }_{128}$ track over drift distance. Second, based on the track position ${ }_{129}$ along the Bragg curve, we expect small changes in the initial ${ }_{130}$ ionization density along the track $(d E / d x)$, a $z$-independent ef-131 fect. We confirm that neither effect impacts our measurement $t_{132}$ of absolute $z$.
We use a simplified model of charge cloud dynamics to de- ${ }^{109}$

ceipt of the trigger. Consequently, the time or $z$ axis of the pixel

Figure 3: Fit to the folded version of the corrected pixel charge $\left(Q_{p x}\right)$ profile shown in Fig. 2 using the profile fit method described in Section 4.3. The Gaussian function (black dotted line) is fitted to the plot points (red open circles), which are placed at the mean $Q$ and $y_{\text {track }}$ positions for each unsaturated TOT layer. Error bars on the points are too small to show. A color version of this figure is available online.

\section{Data and analysis}

Our goal is to demonstrate a measurement of absolute $z$ using the charge cloud topology information provided by the chip. Specifically, we fit a Gaussian function to the corrected pixel charge $Q_{p x}$ versus lateral distance from track center (the charge "profile"). If we know the parameters $A$ and $B$ in Eqn. 2 then the fitted Gaussian width $\sigma$ would uniquely measure absolute $z$ independent from initial track ionization energy. To this end, we focus on a precision determination of the diffusion parameters $A$ and $B$.

Our first task is to verify the model using the embedded alpha sources, then measure the parameters in Eqn. 2, and finally test the performance of the absolute $z$ measurement.

\subsection{Data}

Alpha tracks from the in-situ sources provide an ideal calibration and test of the absolute $z$ determination. We use 80,000 alpha tracks generated during a run with no external sources. We fit these tracks with a linear unweighted least-squares fit to the charge shell and obtain the track polar and azimuthal angles, $\theta$ and $\phi$. Using these track angles and the known position of each alpha source in the detector, we can assign a coordinate in 3-D to each pixel hit in a track; crucially, this allows us to know the "real" $z$ position of each hit, which we label $z_{\text {real }}$.

For each track we rotate to a new "track" coordinate system to look "down the barrel" of the track; the track now lies along the $x_{\text {track }}$ axis, the U-shaped shell opens towards the $z_{\text {track }}$ direction, and the lateral track width dimension is now $y_{\text {track }}$ (see Fig. 1). This coordinate system facilitates easy viewing of the 


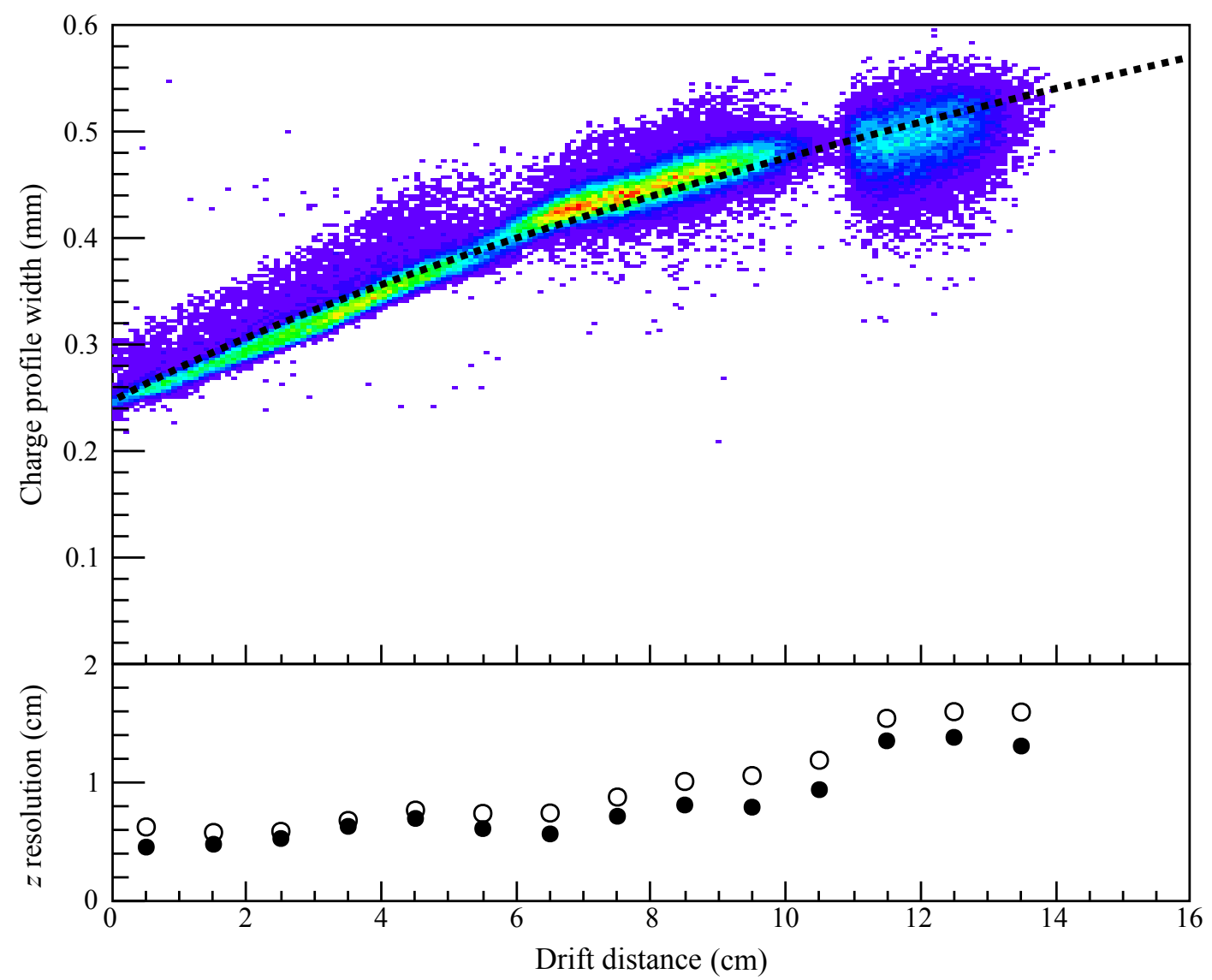

Figure 4: The top plot shows the result of fitting the diffusion equation, Eqn. 2 , to the distribution of charge profile widths versus the drift distance $z_{\text {real }}$, for 8 mm alpha track segments. Data is plotted as a 2-D histogram where the number of track segments per bin are encoded by brightness (color online). The black dotted line shows the fit result. The bottom plot shows the $z$ uncertainty for 8-mm (filled dots) and 2-mm (open dots) segments, defined as the standard deviation in $z$ of the data from the fit curve. A color version of this figure is available online.

track charge profile and shell (Fig. 2). In order to localize $z$-154 dependent effects, we divide each track into $0.8 \mathrm{~cm}$ "segments" ${ }_{155}$ which we evaluate independently for the remainder of the anal-156 ysis.

The alpha segment data constitutes both calibration and val-158 idation data. We use the segments to generate a distribution of 159 cloud shape vs. $z_{\text {real }}$, then extract a functional approximation of 160 that distribution to assign absolute $z$ based on cloud shape. The 161 spread of the distribution around the functional approximation is a measure of the accuracy of the approximation and thus the ${ }_{162}$ absolute $z$ measurement technique.

\subsection{Building charge profiles}

In the track coordinate system, that is, looking down the axis 16 of the track, the charge profile appears as a series of levels cor- ${ }^{-167}$ responding to discrete ToT values, with three main features, la- -168 beled in Fig. 2 (a) for reference. Feature 1 is the predicted Gaus-169 sian charge cross-section. Feature 2 is a cutoff in the Gaussian ${ }_{170}$ tail at a certain charge, due to the chip's threshold setting, here ${ }_{171}$ tuned to a level of $\sim 3,000$ electrons per pixel. Feature 3 is $a_{172}$ flat saturation region corresponding to the high end of the $\mathrm{ToT}_{173}$ scale, tuned to $\sim 25,000$ electrons. Saturation primarily occurs for highly inclined $\left(\theta\right.$ not near $\left.90^{\circ}\right)$ or low- $z$ tracks. The threshold and saturation charges are given by $Q_{p x}\left(\mathrm{ToT}_{\min }\right)$ and $Q_{p x}\left(\mathrm{ToT}_{\max }\right)$, which depend on the track inclination $\theta$ due to the factor $\sin \theta$ in Equation 2.2. Due to the very slow dependence of ionization energy density on distance from the source, the charge density profile is approximately uniform along the track length.

\subsection{Profile fitting}

To fit the charge profile, we find a series of points that trace the Gaussian profile shape and fit a Gaussian function to these points. To achieve this, first we fold the charge profile distribution about the $Q_{p x}$ axis. Second, we identify ToT levels that contain valuable Gaussian shape information; these include unsaturated levels that do not fold over the origin, which we reject by requiring no hits within $10 \mu \mathrm{m}$ of $y_{\text {track }}=0$. Third, we assign one point to each remaining ToT level, with a position fixed to the mean lateral position $y_{\text {track }}$ and charge $Q_{p x}(\mathrm{ToT})$ of all entries in that level. We assign horizontal error bars (uncertainty in the mean lateral position) from the standard mean 
error. Vertical error bars are asymmetric, with height given by222 $\left(Q_{p x}(\mathrm{ToT} \pm 0.5)-Q_{p x}(\mathrm{ToT})\right) /(1 / 2 \sqrt{N})$, that is, half of the dis-223 tance in $Q$ to the next ToT level, divided by $1 / 2 \sqrt{N}$, where $N^{224}$ is the number of entries in the level. We chose this weighting 225 based on fit tests performed on toy Monte Carlo charge profiles.226 Finally, we perform a simple least-squares fit to these points us-227 ing a Gaussian of height $h$ and width $\sigma$, centered on $y_{\text {track }}=0.228$ An example of this fit applied to the charge profile of a flat track229 $\left(\theta=90^{\circ}\right)$ is shown in Fig. 3, where the points are shown with-230 out error bars.

A very small number $(0.6 \%)$ of the segment charge profile232 fits fail, and these are removed. Otherwise, no selections are ap-2зз plied and therefore there is no difference between the precision 234 of the calibration and the accuracy of the subsequent absolute $z^{235}$ prediction

\section{Results}

Our primary result is a least-squares fit, shown in Fig. 4,240 matching Eqn. 2 to the scatterplot of charge profile Gaus-241 sian widths versus known $z$ for $0.8-\mathrm{cm}$ segments selected from ${ }^{242}$ 80,000 alpha tracks. The good quality of this fit validates the physical model. The fit parameters obtained are $A=252 \pm$ $21 \mu \mathrm{m}$ and $B=127 \pm 8 \mu \mathrm{m} / \sqrt{\mathrm{cm}}$. While in our conceptual model $A$ is the readout plane resolution, in practice this fit ${ }_{244}$ parameter is also sensitive to additional $z$-independent contri- ${ }_{245}$ butions to the charge profile Gaussian width, such as electric ${ }_{246}$ field non-uniformity and deviations of the initial charge cloud $_{247}$ from a delta function. $B$ is the diffusion per $\sqrt{\mathrm{cm}}$, in excellent ${ }_{248}$ agreement with the Magboltz prediction [6] of $129 \mu \mathrm{m} / \sqrt{c m}_{249}$ and $124 \mu \mathrm{m} / \sqrt{\mathrm{cm}}$ for the transverse and longitudinal diffusion 250 constants, which are admixed in this measurement.

Now we can use these parameters to predict absolute $z$ for ${ }_{252}$ any segment of ionization charge detected:

$$
z=\frac{\sigma^{2}-A^{2}}{B^{2}}
$$

The absolute $z$ accuracy is measured by the horizontal standard ${ }_{255}^{254}$ deviation of the distribution from the fit curve in Fig. 4. For all256 segments, without any selections on fit quality besides requiring ${ }_{258}^{257}$ that the fit not fail (efficiency 0.994), the absolute $z$ measure ${ }^{259}$ ment accuracy is $0.5-1.5 \mathrm{~cm}$ throughout the full $z$ range. For 260 nuclear recoil tracks, induced by the scattering of neutrons or $^{261}$ Weakly Interacting Massive Particles (WIMPs) in the detector, ${ }^{262}$ the absolute $z$ precision will depend on the length of the track ${ }_{264}^{263}$ To evaluate the effect of small track lengths, we have measured265 the accuracy of this calibration in predicting the absolute $z$ posi-266 tion of $2 \mathrm{~mm}$ alpha track segments. Although a larger number of ${ }_{268}^{267}$ the short-segment fits failed (efficiency 0.82), accuracy is nearly ${ }_{269}^{268}$ as good as with the $8 \mathrm{~mm}$ segments (see Fig. 4). We plan to in-270 vestigate whether this can be easily extended to nuclear recoils $\mathrm{s}^{271}$ next.

\section{Summary and discussion}

We have presented a method for measuring absolute $z$ in TPCs using charge cloud topology information. The key techni- cal features that enable this technique are high spatial resolution of the pixel chip and charge measurement in each pixel. While high spatial resolution alone is enough to measure the diffusion width of tracks, this width also depends strongly on nuisance parameters, such as the detector threshold, gas purity, and the specific ionization of the track. By also utilizing charge information, the technique presented here does not require knowledge of the initial ionization energy and is tolerant of saturation and threshold effects.

It is possible to further optimize the absolute $z$ precision using a lower threshold and higher maximum ToT scale, though gains may be small except for very low $z$. This technique is best used as a live calibration of the absolute $z$ scale since the diffusion parameters may depend on gas quality, gain, drift velocity and other factors and thus may not be stable over time. Embedding one or more sources such as Polonium-210 in the vessel during operation can provide time-dependent calibrations.

The technique used here also provides a way to recover charge lost due to chip threshold and saturation effects, which naturally provides a measurement of gas impurities by way of charge loss over drift distance. This will be reported separately.

\section{Acknowledgements}

We thank Jared Yamaoka and Steven Ross, former Hawaii group members who designed a pixel readout board used in this work. We thank John Kadyk and Maurice Garcia-Sciveres of Lawrence Berkeley National Laboratory for continued support and collaboration, and Kamalu Beamer for assistance during detector construction. We acknowledge support from the U.S. Department of Homeland Security under Award Number 2011DN-077-ARI050-03 and the U.S. Department of Energy under Award Number DE-SC0007852.

\section{References}

[1] D. R. Nygren and J. N. Marx, "The Time Projection Chamber," Phys. Today 31N10, 46 (1978).

[2] D. P. Snowden-Ifft, "Discovery of multiple, ionization-created CS2 anions and a new mode of operation for drift chambers," Rev. Sci. Instrum. 85, 013303 (2014).

[3] E. Daw, J. R. Fox, J. L. Gauvreau, C. Ghag, L. J. Harmon, M. Gold, E. R. Lee and D. Loomba et al., "Spin-Dependent Limits from the DRIFTIId Directional Dark Matter Detector," Astropart. Phys. 35, 397 (2012) [arXiv:1010.3027 [astro-ph.CO]].

[4] D. P. Snowden-Ifft, personal communication, October 2014

[5] S. E. Vahsen, M. T. Hedges, I. Jaegle, S. J. Ross, I. S. Seong, T. N. Thorpe, J. Yamaoka and J. A. Kadyk et al., "3-D Tracking of Nuclear Recoils in a Miniature Time Projection Chamber," arXiv:1407.7013 [physics.ins-det].

[6] All drift velocities used were calculated with the Magboltz software, version 10.0.1, 10. S. F. Biagi, Nucl. Instrum. Meth. A421, 234-240 (1999).

[7] I. Jaegle, S.E. Vahsen, et al., "High Resolution 3-D Tracking in a TPC with Pixel Readout, in preparation (2014).

[8] F. Sauli, "GEM: A new concept for electron amplification in gas detectors," Nucl. Instrum. Meth. A386, 531-534 (1997).

[9] G. Aad, M. Ackers, F. A. Alberti, M. Aleppo, G. Alimonti, J. Alonso, E. C. Anderssen, A. Andreani et al., "ATLAS pixel detector electronics and sensors," JINST 3, P07007 (2008). 